Acta Crystallographica Section F

Structural Biology

Communications

ISSN 2053-230X

Ramya R. Rao and Catherine L. Lawson*

Department of Chemistry and Chemical Biology, Rutgers University, Piscataway, NJ 08854, USA

Correspondence e-mail:

cathy.lawson@rutgers.edu

Received 6 February 2014

Accepted 8 March 2014

PDB reference: catabolite activator protein, $4 \mathrm{ft} 8$

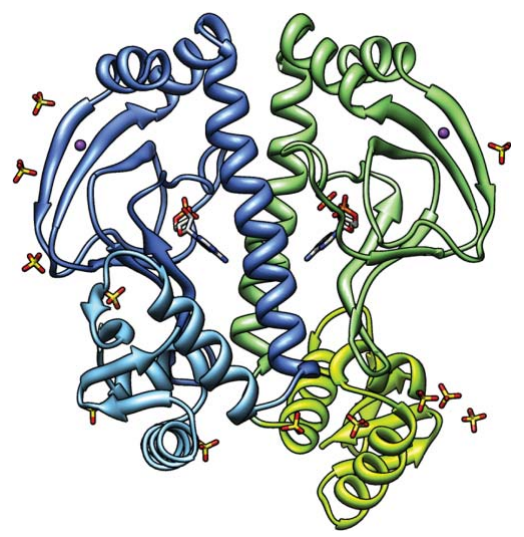

\section{Structure of catabolite activator protein with cobalt(II) and sulfate}

The crystal structure of cyclic AMP-catabolite activator protein (CAP) from Escherichia coli containing cobalt(II) chloride and ammonium sulfate is reported at $1.97 \AA$ resolution. Each of the two CAP subunits in the asymmetric unit binds one cobalt(II) ion, in each case coordinated by N-terminal domain residues His19, His21 and Glu96 plus an additional acidic residue contributed via a crystal contact. The three identified N-terminal domain cobalt-binding residues are part of a region of CAP that is important for transcription activation at class II CAP-dependent promoters. Sulfate anions mediate additional crystal lattice contacts and occupy sites corresponding to DNA backbone phosphate positions in CAP-DNA complex structures.

\section{Introduction}

CAP (catabolite activator protein) is a DNA-binding protein and transcription activator that recruits RNA polymerase to more than 100 genes in Escherichia coli (Busby \& Ebright, 1999; Lawson et al., 2004; Zheng et al., 2004). The CAP dimer consists of two identical 209-residue subunits, each with an $\mathrm{N}$-terminal binding domain for allosteric effector cAMP, and a C-terminal winged helix-turn-helix DNA-binding motif. CAP has three defined 'activating regions', patches of surface-exposed residues that interact with RNA polymerase (RNAP) in specific CAP-dependent promoter classes. Activating region 1 (AR1), located on the $\mathrm{C}$-terminal domain, attracts a flexibly tethered RNAP $\alpha$ subunit $\mathrm{C}$-terminal domain to bind to a DNA site adjacent to CAP (Benoff et al., 2002; Lawson et al., 2004; Hudson et al., 2009). AR2, located on the N-terminal cAMP-binding domain, functions specifically at class II promoters and is predicted to make direct contact with an acidic loop of the RNAP $\alpha^{\mathrm{I}}$ subunit Nterminal domain ( $\alpha \mathrm{NTD}^{\mathrm{I}}$; Niu et al., 1996; Busby \& Ebright, 1999; Lawson et al., 2004). AR3 is also located on the N-terminal domain and is predicted to make direct contact with RNAP $\sigma$ subunit region 4 (Rhodius \& Busby, 2000).

Three distinct crystal forms of native cAMP-bound CAP from $E$. coli have been reported previously: (i) space group $P 2_{1} 2_{1} 2_{1}$ with one dimer in the asymmetric unit (PDB entry 1g6n; McKay et al., 1982; Passner et al., 2000), (ii) space group $P 2_{1}$ with one dimer in the asymmetric unit (PDB entry 4hzf; Rodgers et al., 2013) and (iii) space group $P 1$ with two dimers in the asymmetric unit (PDB entry 2gzw; RIKEN Structural Genomics/Proteomics Initiative, unpublished work). We describe here the structure of cAMP-CAP in a novel fourth crystal form (space group $P 2_{1} 2_{1} 2$ with one dimer in the asymmetric unit) in which cobalt(II) binds to key residues of CAP AR2. In addition, discrete sulfate-anion-binding sites are found near AR2, near the cAMP-binding site and at positions analogous to DNA phosphate positions in CAP-DNA complexes. This crystal form of cAMP-CAP was fortuitously obtained during efforts to structurally characterize a 2:1 CAP dimer-DNA complex under conditions that apparently disrupted the protein-DNA interaction (Rao, 2011). 


\section{Materials and methods}

\subsection{Preparation of CAP}

Full-length CAP (209 residues) was expressed and purified by cAMP-affinity chromatography, as described in Zhang et al. (1991), and further purified on a Heparin 16/10 FF column (GE Healthcare). CAP was then concentrated and buffer-exchanged to approximately $360 \mu M$ dimer (approximately $18 \mathrm{mg} \mathrm{ml}^{-1}$ ) in $20 \mathrm{~m} M$ Tris $\mathrm{pH} \mathrm{8.0,}$ $0.1 \mathrm{~m} M$ EDTA, $1 \mathrm{~m} M$ DTT, $200 \mathrm{~m} M \mathrm{NaCl}, 0.02 \%(w / v) \mathrm{NaN}_{3}$. The protein concentration was determined using a Bradford assay. CAP purity was estimated to be $>95 \%$.

\subsection{Crystallization and data collection}

A 71 bp DNA duplex containing two tandem CAP sites separated by 32 base pairs was designed according to a promoter sequence described by Tebbutt et al. (2002) and was assembled using three oligonucleotides (Rao, 2011, p. 11). DNA oligonucleotides were obtained from IDT and were annealed to form duplex DNA using a standard laboratory procedure (Locasale et al., 2009). The CAPDNA complex used in crystallization trials consisted of $0.1 \mathrm{~m} M$ CAP dimer, $0.05 \mathrm{~m} M$ DNA duplex, $0.4 \mathrm{~m} M$ cyclic AMP.

Crystallization screening was by hanging-drop vapor diffusion using Hampton Research crystal screens and pre-greased VDX plates. Optimization of Crystal Screen 2 condition No. 25 yielded ruby-red-colored crystals that appeared within $3 \mathrm{~d}$ at $20^{\circ} \mathrm{C}$ in $0.01 \mathrm{M}$ $\mathrm{CoCl}_{2} \cdot 6 \mathrm{H}_{2} \mathrm{O}, 0.1 \mathrm{M}$ MES monohydrate $\mathrm{pH}$ 6.5, $2.5 \mathrm{M}$ ammonium sulfate.

Crystals were mounted on nylon loops and flash-cooled in liquid nitrogen after being submerged briefly in a cryoprotectant solution comprised of $66.5 \%(w / w)$ Paratone-N, $28.5 \%(w / w)$ paraffin oil, $5 \%(w / w)$ glycerol. X-ray diffraction data were collected on beamline
$\mathrm{X} 8 \mathrm{C}$ at the National Synchrotron Light Source at Brookhaven National Laboratory to $1.97 \AA$ resolution.

\subsection{Structure determination and refinement}

The diffraction data were processed and scaled assuming primitive orthorhombic lattice symmetry using the $H K L-2000$ package (Otwinowski \& Minor, 1997). Molecular-replacement search models were prepared using selected coordinates from PDB entry 1zrc (Napoli et al., 2006). Molecular-replacement trials were carried out in all possible primitive orthorhombic space groups using Phaser (McCoy et al., 2007). An unambiguous solution was obtained. The Nterminal domain dimer of CAP (residues 1-136) was positioned first, yielding a $Z$-score of 18.7 in space group $P 22_{1} 2_{1}$; the next highest $Z$ score was 10.0 in space group $P 22_{1} 2$. The two C-terminal domains (residues 137-209) were located in subsequent Phaser runs. After the molecular replacement, the model coordinates and diffraction data $h k l$ Miller indices were transformed to the standard $P 2_{1} 2_{1} 2$ setting.

Noncrystallographic twofold symmetry observed in the self-rotation function and Matthews coefficient analysis (Kantardjieff \& Rupp, 2003; Matthews, 1968) provided additional support for the assignment of one complete CAP dimer in the crystal asymmetric unit. Upon inspection of crystal packing and likelihood-weighted electron density, it became evident that the crystal structure did not contain DNA.

Crystallographic refinement was initially performed using REFMAC5 (Murshudov et al., 2011) and in the final stages using PHENIX (Adams et al., 2010). Noncrystallographic symmetry restraints for CAP N-terminal and C-terminal domains were maintained and separately defined. The model was inspected between refinement runs and manually adjusted to fit likelihood-weighted electron-density maps using Coot (Emsley \& Cowtan, 2004). Solvent
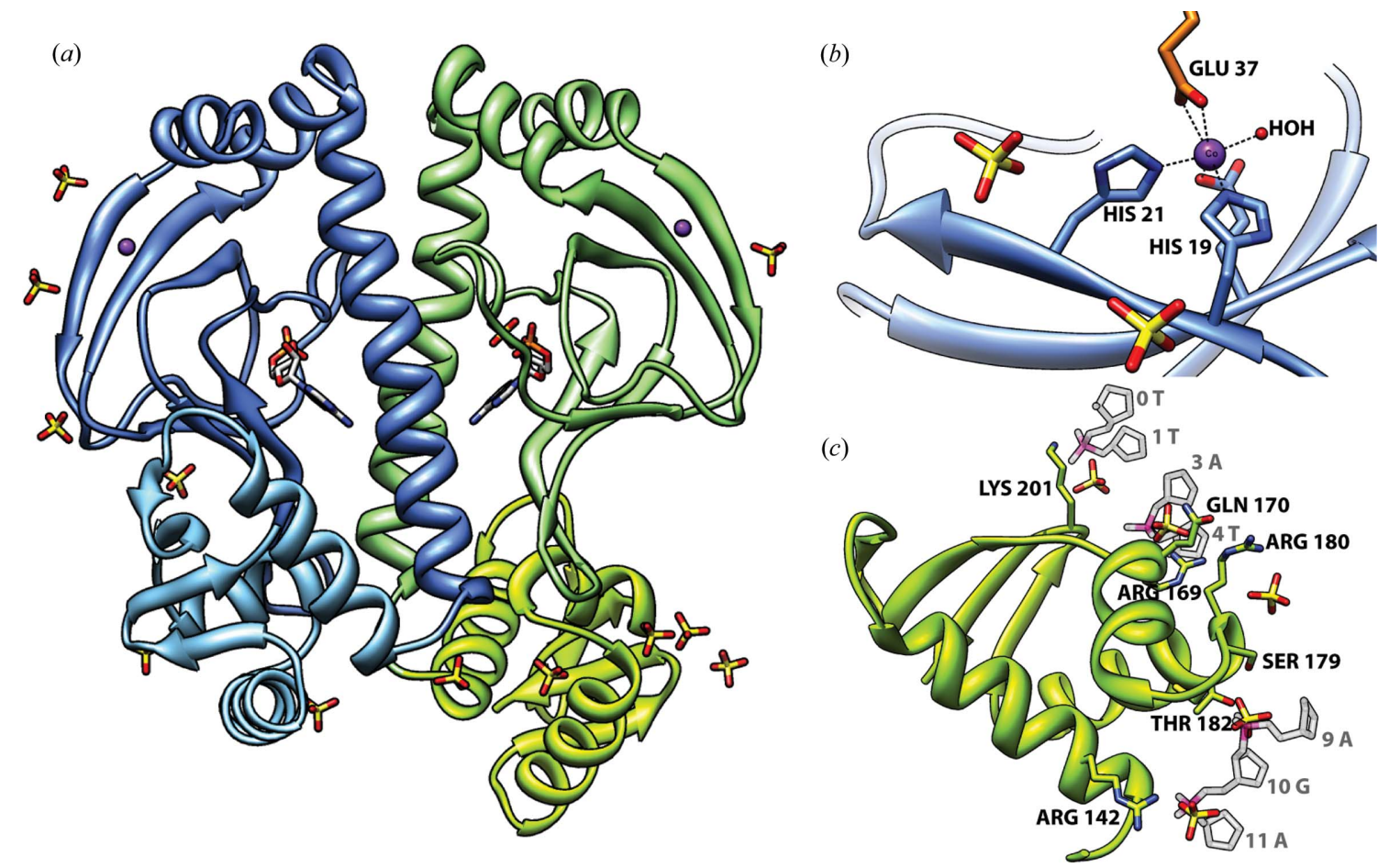

Figure 1

cAMP-CAP dimer with bound cobalt(II) and sulfate ligands (CAP subunits, blue/light-blue and green/lime ribbons; cAMP and sulfate, stick representation with atom-type colors; $\mathrm{Co}^{2+}$, purple spheres). (a) Crystal asymmetric unit composed of one cAMP-CAP dimer, two $\mathrm{Co}^{2+}$ ions and 13 sulfate ligands. $(b) \mathrm{Co}^{2+}$ coordination in subunit $A$ (crystal lattice neighbor shown at top in orange). (c) C-terminal domain of subunit $B$ shown with bound sulfates and selected DNA sugar-phosphate backbone fragments from a superimposed CAP-DNA structure [PDB entry 1zrc (Napoli et al., 2006), translucent grey/pink sticks]. DNA numbering follows Fig. 1 of Lawson $e t$ al. (2004). 
Table 1

Crystal, data collection and refinement statistics and model information.

Values in parentheses are for the outer resolution shell.

\begin{tabular}{ll}
\hline Crystal data & \\
Crystal system, space group & Orthorhombic, $P 2_{1} 2_{1} 2$ \\
Unit-cell parameters $(\AA)$ & $a=104.14, b=108.45, c=44.32$ \\
No. of CAP dimers in unit cell $Z$ & 4 \\
Matthews coefficient $V_{\mathrm{M}}\left(\AA^{3} \mathrm{Da}^{-1}\right)$ & 2.50 \\
Solvent content $(\%)$ & 50.8 \\
Data collection & \\
Diffraction source & Beamline X8C, NSLS \\
Wavelength $(\AA)$ & 1.1 \\
Detector & ADSC Quantum 4 \\
Temperature $\left({ }^{\circ} \mathrm{C}\right)$ & -173 \\
Resolution range $(\AA)$ & $50.0-1.97(2.03-1.97)$ \\
No. of unique reflections & $35645(3280)$ \\
No. of observed reflections & 181462 \\
Completeness $(\%)$ & 97.1 \\
Multiplicity & $5.1(4.2)$ \\
$\langle I / \sigma(I)\rangle$ & 9.5 \\
$R_{\text {merge }}$ & $0.088(0.778)$ \\
Data-processing software & $D E N Z O, S C A L E P A C K$ \\
Refinement & \\
Refinement software & phenix.refine, PHENIX \\
$\sigma$ cutoff & $F>1.340 \sigma(F)$ \\
Resolution range $(\AA)$ & $40.78-1.97(2.02-1.97)$ \\
No. of reflections used in refinement & 33823 \\
No. of reflections above $\sigma$ cutoff & 35597 \\
Final overall $R$ factor & 0.194 \\
Atomic displacement model & Individual isotropic $B$ factors \\
Overall average $B$ factor $\left(\AA^{2}\right)$ & 35.5 \\
No. of protein atoms & 3268 \\
R.m.s.d., bond lengths $(\AA)$ & 0.010 \\
R.m.s.d., bond angles $\left({ }^{\circ}\right)$ & 1.007 \\
No. of ligand atoms & 116 \\
No. of solvent atoms & 410 \\
Total No. of atoms & 3794 \\
No. of refined parameters & 15176 \\
NCS restraints & Removed in final refinement cycles \\
Bulk-solvent model & Flat; $B_{\text {sol }}=58.65 \AA^{2}, k_{\text {sol }}=0.37$ e $\AA^{-3}$ \\
Final $R_{\text {work }}$ & $0.194(0.286)$ \\
No. of reflections for $R_{\text {free }}$ & 1774 \\
Final $R_{\text {free }}$ & $0.229(0.324)$ \\
\hline & \\
\hline &
\end{tabular}

atoms were initially added manually using conservative criteria; in the final refinement stages, the phenix.refine water-picking protocol was used with default settings. Cobalt(II) and sulfate ligands were manually placed in positions of strong electron density at the protein surface that could not be satisfactorily modeled as solvent owing to density strength, shape and local stereochemistry criteria.

\subsection{Structure analysis}

The quality and stereochemistry of the model and fit to electron density were evaluated using validation tools in Coot. The CCP4 suite (Winn et al., 2011) programs CONTACT and SUPERPOSE were used to evaluate contacts between protein and ligands and to perform superposition calculations. Molecular-graphics images were created using UCSF Chimera (Pettersen et al., 2004).

\section{Results}

Our efforts to characterize a CAP-DNA complex fortuitously yielded a crystal structure for CAMP-CAP without DNA in a novel crystal form in which cobalt and sulfate ligands are bound to CAP. This structure is shown schematically in Fig. 1(a). The crystal asymmetric unit contains one CAP dimer (residues 7-207 of chain $A$ and 7-206 of chain $B$ were modeled out of 210 total residues per subunit), with cAMP ligands bound as expected within the N-terminal domain (Berman et al., 2005), two cobalt(II) cations, 13 sulfate anions and 420 solvent atoms.
The main-chain torsion angles of most residues (96\%) lie in preferred regions of the Ramachandran plot, with the remainder (4\%) in the allowed regions. The final $R$ factor is $19.4 \%$ and $R_{\text {free }}$ is $22.9 \%$ (Table 1 ).

The conformation of the CAP dimer in this new crystal form is essentially identical to the conformation observed in earlier structure reports for cAMP-CAP (McKay et al., 1982; Passner et al., 2000), with two cAMP-binding N-terminal domains related by local twofold rotational $(C 2)$ symmetry, and flexibly tethered DNA-binding Cterminal domains that adopt two distinct positions relative to the $\mathrm{N}$ terminal domains. Superposition of all $400 \mathrm{C}^{\alpha}$ atoms in common with the CAP dimer of PDB entry 1g6n (Passner et al., 2000) yields a rootmean-square deviation (r.m.s.d.) of only $1.4 \AA$ A. The 'closed' conformation of subunit $A$ (Fig. 1a, blue/light-blue ribbon) closely resembles the conformation of DNA-bound CAP (Schultz et al., 1991; Parkinson et al., 1996). In the 'open' conformation of subunit $B$ (Fig. $1 a$, green/lime ribbon), the C-terminal domain is rotated by $40^{\circ}$ and translated by $15 \AA$ relative to the closed conformation.

The full $A$ and $B$ subunits superimpose poorly, owing to their observed conformational difference (r.m.s.d. of 3.9 $\AA$ ). However, the individual $\mathrm{N}$ - and $\mathrm{C}$-terminal domains from each subunit readily superimpose (r.m.s.d.s of 1.1 and $0.4 \AA$, respectively). The $\mathrm{N}$ - and Cterminal domains of the new structure also readily superimpose with previously determined cAMP-CAP crystal structure domains [r.m.s.d.s of $\leq 0.9$ and $\leq 1.5 \AA$, respectively, from PDB entries $1 \mathrm{~g} 6 \mathrm{n}$ (Passner et al., 2000), 4hzf (Rodgers et al., 2013) and 2gzw (RIKEN Structural Genomics/Proteomics Initiative, unpublished work)].

The two $\mathrm{Co}^{2+}$ ions bound to the CAP dimer have chemically similar environments on each $\mathrm{N}$-terminal domain, even though the positions are not crystallographically equivalent (Figs. $1 a$ and $1 b$, purple spheres). Each has approximate octahedral coordination (Fig. $1 b$ ), with three liganding atoms contributed by N-terminal domain residues His19 $\left(\mathrm{N}^{\varepsilon 2}\right)$, His21 $\left(\mathrm{N}^{\varepsilon 2}\right)$ and Glu96 $\left(\mathrm{O}^{\varepsilon 1}\right.$ or $\left.\mathrm{O}^{\varepsilon 2}\right)$. In each case, a neighboring CAP dimer related by crystal symmetry contributes two additional ligands from residue Glu37 $\left(\mathrm{O}^{\varepsilon 1}\right.$ and $\left.\mathrm{O}^{\varepsilon 2}\right)$. Octahedral coordination is completed at each site by a solvent water $\mathrm{O}$ atom. The average $\mathrm{Co}^{2+}$-to-coordinating-atom bond length is 2.22 $\pm 0.12 \AA$ (12 measurements; range $2.03-2.44 \AA$ ) and the average atom-to- $\mathrm{Co}^{2+}$-to-neighbor-atom bond angle is $92.0 \pm 11.0^{\circ}(21$ measurements; range $57.0-113.1^{\circ}$ ).

13 sulfate anions were also identified at positions adjacent to the protein surface (Fig. 1; sulfates are shown in stick representation with sulfur in yellow and oxygen in red). Six of these mediate proteinsulfate-protein contacts between the CAP dimer and crystal lattice neighbors (Table 2).

\section{Discussion}

The identification of $\mathrm{Co}^{2+}$ tightly bound to the CAP AR2 region confirms the hypothesis originally put forward by Wickstrum \& Egan (2002) that CAP can bind metals and, in addition, that residue His19 is involved in the metal-binding activity. Their hypothesis was based on the observation that CAP can effectively bind to $\mathrm{Ni}^{2+}$-charged chromatography media, with wild-type CAP eluting at a substantially higher concentration of imidazole than the CAP mutant His19Ala.

The three $\mathrm{N}$-terminal domain $\mathrm{Co}^{2+}$-binding residues (His19, His 21 and Glu96) constitute a major subset of the four residues previously identified to form CAP activating region 2 (AR2), which participates in transcription activation specifically at class II CAP-dependent promoters (Niu et al., 1996). In vivo, His19Ala, His21Ala and Lys101Ala mutations each result in an approximately fivefold 
Table 2

cAMP-CAP dimer sulfate environments.

\begin{tabular}{|c|c|c|}
\hline Crystal asymmetric unit environment & $\begin{array}{l}\text { Crystal lattice } \\
\text { neighbors }\end{array}$ & CAP region \\
\hline His $21 A \mathrm{~N}^{\delta 1}$, Lys $22 A \mathrm{~N}$ & Lys $35 B \mathrm{~N}^{\zeta}$ & AR2 \\
\hline His $21 B \mathrm{~N}^{\delta 1}$, Lys $22 B \mathrm{~N}$ & Lys $35 A \mathrm{~N}^{\zeta}$ & AR2 \\
\hline Ile $20 A \mathrm{~N}$ & & AR2 \\
\hline $\begin{array}{l}\text { Asp68B } \mathrm{O}^{\delta 1}, \mathrm{Gln} 119 B \mathrm{O}^{\varepsilon 1}, \operatorname{Arg} 122 B \mathrm{~N}^{\varepsilon}, \mathrm{N}^{\eta 2}, \\
\quad \operatorname{Arg} 123 B \mathrm{~N}^{\varepsilon}, \mathrm{N}^{\eta 2}\end{array}$ & & cAMP site \\
\hline His $31 A \mathrm{~N}^{\delta 1}, \operatorname{Trp} 85 \mathrm{~A} \mathrm{~N} \mathrm{~N}^{\varepsilon 1}$ & His $31 B \mathrm{~N}^{\delta 1}, \operatorname{Tr} p 85 \mathrm{~B} \mathrm{~N}^{\varepsilon 1}$ & cAMP site \\
\hline Glu81A $\mathrm{O}^{\varepsilon 2}$ & Thr $28 B \mathrm{~N}$ & cAMP site \\
\hline \multicolumn{3}{|l|}{ Ala91 $A \mathrm{~N}$} \\
\hline Val139B N, Arg142B $\mathrm{N}^{\eta 1}$ & & DNA $\mathrm{PO}_{4}$ \\
\hline $\operatorname{Arg} 169 A \mathrm{~N}, \mathrm{G} \ln 170 A \mathrm{~N}$ & & DNA $\mathrm{PO}_{4}$ \\
\hline Thr168B $\mathrm{O}^{\gamma 1}, \operatorname{Arg} 169 B \mathrm{~N}, \mathrm{Gln} 170 B \mathrm{~N}$ & & DNA $\mathrm{PO}_{4}$ \\
\hline Lys $57 B$ N $\zeta$, Ser $179 B$ N, $\mathrm{O}^{\gamma}$, Thr182B $\mathrm{O}^{\gamma 1}$ & & DNA $\mathrm{PO}_{4}$ \\
\hline $\operatorname{Arg} 180 B \mathrm{~N}, \mathrm{~N}^{\eta 1}, \mathrm{~N}^{\eta 2}$ & & DNA binding \\
\hline Lys $201 B$ N & Ty $206 A \mathrm{O}^{\eta}$ & DNA $\mathrm{PO}_{4}$ \\
\hline
\end{tabular}

reduction in class II transcription activation, while Glu96Ala mutation results in an approximately threefold improvement; these same mutations have no effect on class I transcription activation (Niu et al., 1996). At class II promoters, CAP binds to a site $\sim-45$ bp upstream of the transcription start site, and CAP-AR2 is predicted based on cross-linking analysis to contact a highly acidic loop in the N-terminal domain of the RNAP $\alpha^{\mathrm{I}}$ subunit with sequence EEDE (residues 162165; Niu et al., 1996; Lawson et al., 2004).

In the crystal structure reported here, $\mathrm{Co}^{2+}$-metal-binding coordination by the three AR2 residues is completed through an acidic residue provided in 'trans' through a crystal contact. Although no specific metal requirement has been reported for CAP class II CAPdependent transcription activation, our structure raises the possibility that the local environment of AR2 in contact with the acidic loop of the RNAP $\alpha^{\mathrm{I}}$ subunit could involve or accommodate metal binding.

Several sulfates in the crystal structure adopt positions that correspond to phosphate sites of the DNA backbone in CAP-DNA crystals (Table 2, Fig. 1c). The DNA in the CAP dimer-DNA complexes is strongly bent and electrostatic interactions are thought to play an important role in DNA bending (Schultz et al., 1991; Gartenberg \& Crothers, 1988; Kapanidis et al., 2001); our structure suggests that the CAP C-terminal domains have an intrinsic capability to bind to DNA half-sites prior to adoption by CAP of its fully bent DNA binding conformation.

We thank Samuel Lara-González and Brian P. Hudson for assistance with material preparation and data collection. The National Synchrotron Light Source is supported by the US Department of
Energy, Office of Basic Energy Sciences. This work was supported by National Institutes of Health grant No. GM021589 to CLL.

\section{References}

Adams, P. D. et al. (2010). Acta Cryst. D66, 213-221.

Benoff, B., Yang, H., Lawson, C. L., Parkinson, G., Liu, J., Blatter, E., Ebright, Y. W., Berman, H. M. \& Ebright, R. H. (2002). Science, 297, 1562-1566.

Berman, H. M., Ten Eyck, L. F., Goodsell, D. S., Haste, N. M., Kornev, A. \& Taylor, S. S. (2005). Proc. Natl Acad. Sci. USA, 102, 45-50.

Busby, S. \& Ebright, R. H. (1999). J. Mol. Biol. 293, 199-213.

Emsley, P. \& Cowtan, K. (2004). Acta Cryst. D60, 2126-2132.

Gartenberg, M. R. \& Crothers, D. M. (1988). Nature (London), 333, 824-829.

Hudson, B. P., Quispe, J., Lara-González, S., Kim, Y., Berman, H. M., Arnold, E., Ebright, R. H. \& Lawson, C. L. (2009). Proc. Natl Acad. Sci. USA, 106, 19830-19835.

Kantardjieff, K. A. \& Rupp, B. (2003). Protein Sci. 12, 1865-1871.

Kapanidis, A. N., Ebright, Y. W., Ludescher, R. D., Chan, S. \& Ebright, R. H. (2001). J. Mol. Biol. 312, 453-468.

Lawson, C. L., Swigon, D., Murakami, K. S., Darst, S. A., Berman, H. M. \& Ebright, R. H. (2004). Curr. Opin. Struct. Biol. 14, 10-20.

Locasale, J. W., Napoli, A. A., Chen, S., Berman, H. M. \& Lawson, C. L. (2009). J. Mol. Biol. 386, 1054-1065.

Matthews, B. W. (1968). J. Mol. Biol. 33, 491-497.

McCoy, A. J., Grosse-Kunstleve, R. W., Adams, P. D., Winn, M. D., Storoni, L. C. \& Read, R. J. (2007). J. Appl. Cryst. 40, 658-674.

McKay, D. B., Weber, I. T. \& Steitz, T. A. (1982). J. Biol. Chem. 257, 9518-9524.

Murshudov, G. N., Skubák, P., Lebedev, A. A., Pannu, N. S., Steiner, R. A., Nicholls, R. A., Winn, M. D., Long, F. \& Vagin, A. A. (2011). Acta Cryst. D67, 355-367.

Napoli, A. A., Lawson, C. L., Ebright, R. H. \& Berman, H. M. (2006). J. Mol. Biol. 357, 173-183.

Niu, W., Kim, Y., Tau, G., Heyduk, T. \& Ebright, R. H. (1996). Cell, 87, 1123 1134.

Otwinowski, Z. \& Minor, W. (1997). Methods Enzymol. 276, 307-326.

Parkinson, G., Wilson, C., Gunasekera, A., Ebright, Y. W., Ebright, R. H., Ebright, R. E. \& Berman, H. M. (1996). J. Mol. Biol. 260, 395-408.

Passner, J. M., Schultz, S. C. \& Steitz, T. A. (2000). J. Mol. Biol. 304, 847-859.

Pettersen, E. F., Goddard, T. D., Huang, C. C., Couch, G. S., Greenblatt, D. M., Meng, E. C. \& Ferrin, T. E. (2004). J. Comput. Chem. 25, 1605-1612.

Rao, R. R. (2011). Masters thesis, Rutgers University, USA.

Rhodius, V. A. \& Busby, S. J. (2000). J. Mol. Biol. 299, 311-324.

Rodgers, T. L., Townsend, P. D., Burnell, D., Jones, M. L., Richards, S. A., McLeish, T. C., Pohl, E., Wilson, M. R. \& Cann, M. J. (2013). PLoS Biol. 11, e1001651.

Schultz, S. C., Shields, G. C. \& Steitz, T. A. (1991). Science, 253, 1001-1007.

Tebbutt, J., Rhodius, V. A., Webster, C. L. \& Busby, S. J. (2002). FEMS Microbiol. Lett. 210, 55-60.

Wickstrum, J. R. \& Egan, S. M. (2002). Biotechniques, 33, 728-730.

Winn, M. D. et al. (2011). Acta Cryst. D67, 235-242.

Zhang, X., Gunasekera, A., Ebright, Y. W. \& Ebright, R. H. (1991). J. Biomol. Struct. Dyn. 9, 463-473.

Zheng, D., Constantinidou, C., Hobman, J. L. \& Minchin, S. D. (2004). Nucleic Acids Res. 32, 5874-5893. 